\title{
Application of $\mu$-FTIR-SR Spectroscopy to Prostate Tissue Analysis
}

\author{
W.M. Kwiatek ${ }^{a, *}$, C. Paluszkiewicz ${ }^{b}$, A. Banaśs ${ }^{a}$, A. Kisiel $^{c}$, M. PodgórczyK $^{a}$, \\ A. Marcelli ${ }^{d}$, M. Cestelli Guidi ${ }^{d, e}$ And M. PiCcinini ${ }^{d, e}$ \\ ${ }^{a}$ Institute of Nuclear Physics, PAS, Radzikowskiego 152, 31-342 Kraków, Poland \\ ${ }^{b}$ AGH University of Science and Technology, al. Mickiewicza 30, 30-059 Kraków, Poland \\ ${ }^{c}$ Institute of Physics, Jagiellonian University, Reymonta 4, 30-059 Kraków, Poland \\ ${ }^{d}$ INFN - Laboratori Nazionali di Frascati, Via E. Fermi 40, I-00044 Frascati (Rome), Italy \\ 'Universita' Roma Tre, Dip. Scienze Geologiche, L. go S. Leonardo Murialdo 1, 00146 Rome, Italy \\ The infrared spectromicroscopy is a quite recent technique rapidly developing thanks to the availability of \\ new instruments and new brilliant synchrotron radiation sources in different areas and in particular to biomedical \\ researches. In order to achieve a diffraction limited spatial resolution in tissue samples, we performed experiments \\ at SINBAD, the synchrotron infrared beamline of the Laboratori Nazionali di Frascati. We characterized the \\ chemical composition of prostate tissue samples taken from patients affected by prostate cancer disease. Different \\ sizes of the pinholes were considered for the measurements. In the case of prostate tissue sections the results \\ show the possibility to determine the intensity ratio of the $\mathrm{CH}_{2}$ and $\mathrm{CH}_{3}$ bands set at $2930 \mathrm{~cm}^{-1}$ and $2960 \mathrm{~cm}^{-1}$, \\ respectively. Experiments were also performed with a pinhole of $5 \mu \mathrm{m}$ of diameter and the differences in both \\ histological and chemical compositions of such samples were determined.
}

PACS numbers: 87.64.km, 87.64.kp, 87.64.Ee, 87.85.jf

\section{Introduction}

The microscale analysis on biomedical samples became very popular since it can give the information about local chemical structure and enables the analysis of chemical compounds. The use of synchrotron radiation based techniques in such analysis gives to researcher a possibility of obtaining information with good signal-to-noise $(\mathrm{S} / \mathrm{N})$ ratio due to the high brilliance of synchrotron radiation. Application of Fourier transform infrared spectroscopy (FTIR) may investigate molecular bonds present in a sample and synchrotron radiation FTIR (SR-FTIR) $\mu$-spectroscopy is the first step to identify structures at subcellular level [1-8].

Molecular structure and function are strongly correlated. This aspect is particularly relevant in the case of proteins, which play important roles in cells biochemistry. Changes of structure may be easily detected in an IR spectrum and a cellular molecular marker may in fact be used to address a pathological status of tissue. Furthermore, comparison of spectra between healthy and cancerous tissue may improve understanding of pathogenesis of morbid process.

Knowledge about biochemistry of cells can be achieved only with an analysis at the microlevel, therefore the au-

* corresponding author; e-mail: Wojciech.Kwiatek@ifj.edu.pl thors made an effort to identify the smallest illumination, e.g. a microbeam size capable to collect spectral data with a $\mathrm{S} / \mathrm{N}$ ratio in order to identify characteristic IR bands and to follow the behavior of specific band intensity ratios in different samples.

Conventional FTIR spectroscopical techniques have been already applied to biomedical samples and it is now well established that typical absorption bands observed in these types of samples are:

- the amide I band, detected around $1650 \mathrm{~cm}^{-1}$ is given by the contribution of the $\nu(\mathrm{C}=\mathrm{O})$ stretching vibrations of protein amide bonds;

- the amide II band, detected around $1549 \mathrm{~cm}^{-1}$ is given by the combination of the $\delta(\mathrm{N}-\mathrm{H})$ bending and the $\nu(\mathrm{C}-\mathrm{N})$ stretching vibrations of the amide bonds;

- the $\nu(\mathrm{C}=\mathrm{O})$ stretching of lipids at about $1740 \mathrm{~cm}^{-1}$;

- the asymmetric $\nu_{\text {as }}\left(\mathrm{PO}_{2}^{-}\right)$stretching of nucleic acids and phospholipids near $1225 \mathrm{~cm}^{-1}$ [9].

In our case we paid special attention to the bands in the wave number region from 4000 to $2000 \mathrm{~cm}^{-1}$. In this range one may also observe bands related to lipid and protein bonds such as $\nu_{\text {as }}\left(\mathrm{CH}_{2}\right.$ and $\left.\mathrm{CH}_{3}\right)$ and $\nu_{\mathrm{s}}\left(\mathrm{CH}_{2}\right.$ and $\left.\mathrm{CH}_{3}\right)$, a choice imposed by the sample preparation 
procedure. In this region the substrate (Mylar foil) on which the tissue sections were placed has no intense characteristic bands $[9,10]$. There are only very weak bands which have no influence on the data analysis.

Cancer is certainly one of the major civilization diseases since the last century and nowadays among the different cancer pathologies, the prostate cancer is one of the most common neoplasm man pathologies. Also for this reason we decided to start a FTIR $\mu$-spectroscopy research on prostate tissue samples.

\section{Materials and methods}

\subsection{Sample preparation}

Samples were obtained by patients affected by prostate cancer diseases and by patients being diagnosed as benign prostate hyperplasia $(\mathrm{BPH})$. Tissues were cut into sections of a thickness of $15 \mu \mathrm{m}$ using a cryomicrotome. The same sections were used for both FTIR analysis and histopathological examination. For FTIR analysis sections were deposited on $2.5 \mu \mathrm{m}$ thick Mylar foils while for histopathological examination sections were mounted on a microscopic glass and stained with hematoxylin and eosin. For staining the adjacent sections were used [11]. The choice of Mylar as the support instead of other typical IR transmitting material such as $\mathrm{KBr}$ follows the use of the same substrate for trace elemental analysis and $\mathrm{X}$-ray absorption near-edge structure (XANES) experiments performed on the same sections at the same spots of the following FTIR experiments [12-14]. Actually, the FTIR spectrum of a Mylar foil contains very weak bands in $4000-2000 \mathrm{~cm}^{-1}$ region [6]. These contributions may be neglected and IR spectral analysis may be performed in this wave number region.

\subsection{Experiment}

Experiments were performed in air with a FTIR system (BRUKER Equinox 55 spectrometer equipped with an IRscope II microscope) available at the Laboratori Nazionali di Frascati of the INFN (Italy) at the Synchrotron INfrared Beamline At DA $\Phi$ NE (SINBAD) beam line [15] in parasitic condition. Spectra taken by SR-FTIR system were measured with different redundant aperture from $250 \mu \mathrm{m} \times 250 \mu \mathrm{m}$ down to $20 \mu \mathrm{m} \times 20 \mu \mathrm{m}$ with $8 \mathrm{~cm}^{-1}$ resolution in transmission mode in the frequency region from $4000 \mathrm{~cm}^{-1}$ to $700 \mathrm{~cm}^{-1}$. In addition, some spectra were measured with different circular pinhole placed on the sample. Each spectrum was collected within about $20 \mathrm{~min}$, e.g., the time between two consecutive injections, a time sufficient to collect about $10^{3}$ scans per point. The BRUKER IRscope II microscope was equipped with a mercury cadmium telluride (MCT) detector of Infrared Labs of $250 \mu \mathrm{m}$ size and an $x y$-stage with a micrometer resolution. A video camera enabled to store optical images of the sample investigated area. Special care was taken to perform experiments at subcellular spatial resolution.

\section{Results}

Trying to achieve resolution at subcellular level, we performed at the SINBAD beam line three independent experiments. Acquisitions were performed on specific sample areas where earlier histopathological examination using a stained adjacent section of the same sample addressed interesting issues. We first started experiments on a cancerous prostate tissue focusing in the spectra on IR bands in the region from 3600 to $2800 \mathrm{~cm}^{-1}$. For each point we collected three spectra of 50 scans each. To obtain more reliable data we averaged the three spectra and the same procedure was applied to the reference point. Absorbance spectra vs. wave number are compared in Fig. 1 for different apertures larger than the diffraction limit (i.e., 3-6 $\mu \mathrm{m}$ in the range $2000-4000 \mathrm{~cm}^{-1}$ ) trying to discriminate molecular information from areas comparable or larger than the cellular size in the same points of a cancerous tissue sample. As one can see, two main peaks occur at about $3300 \mathrm{~cm}^{-1}$ and $2900 \mathrm{~cm}^{-1}$. Each spectrum was normalized first for a base line in the region from $3600 \mathrm{~cm}^{-1}$ to $2700 \mathrm{~cm}^{-1}$, then spectra were also normalized to the number of incident photons.

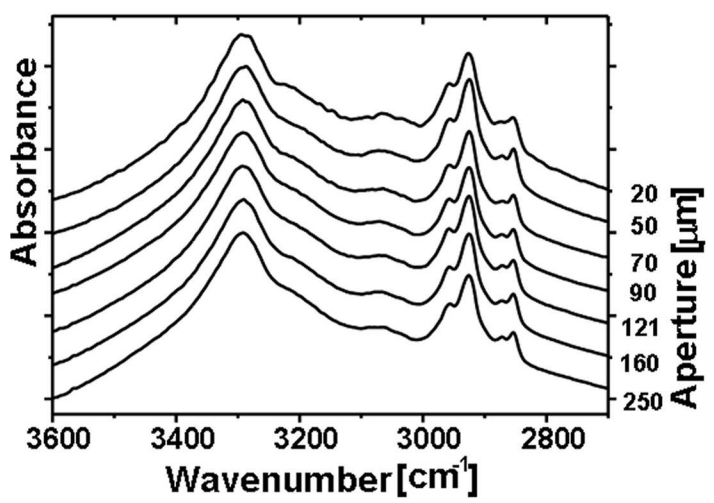

Fig. 1. SR-FTIR spectra collected with a BRUKER IRscope II microscope with different apertures on the same region of a cancerous tissue.

Additional relevant chemical bands associated to the $\mathrm{CH}_{2}$ and $\mathrm{CH}_{3}$ stretching vibrations were detected at $2930 \mathrm{~cm}^{-1}$ and $2960 \mathrm{~cm}^{-1}$, respectively. The behavior presented on a separate graph in Fig. 2 shows that the $\mathrm{CH}_{2}$ to $\mathrm{CH}_{3}$ bands intensity ratio is almost constant or slightly increases following the decrease in the aperture size. Values are greater than $1.32 \pm 0.12$ except for the last point measured with the aperture of $20 \mu \mathrm{m} \times 20 \mu \mathrm{m}$, probably because it is the result of a collection from a limited tissue area containing a very few cancerous cells. Differences can be certainly ascribed to more or less homogeneous distribution of healthy and unhealthy cells in the different surface probed. From full aperture to $20 \mu \mathrm{m} \times 20 \mu \mathrm{m}$ the area decreases by a factor more than 150 .

A second set of experiments was performed on a hyperplastic prostate tissue trying to resolve different tissue 


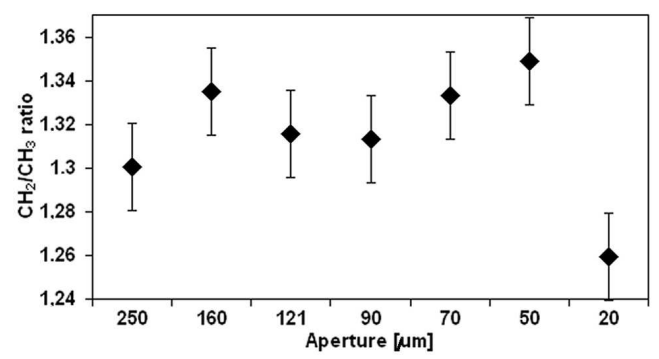

Fig. 2. The $\mathrm{CH}_{2} / \mathrm{CH}_{3}$ intensity ratio of the spectra showed in Fig. 1 with different aperture sizes.

regions with the available microscope experimental setup. In these experiments we sum six spectra, each spectrum was collected with 1000 scans, and the final spectrum was the result of an average of them. The six spectra were collected at the same sample point at the spectral resolution of $8 \mathrm{~cm}^{-1}$. Because the smallest aperture available was $20 \mu \mathrm{m} \times 20 \mu \mathrm{m}$ we used a pinhole of $5 \mu \mathrm{m}$ in diameter and placed it on the surface of the sample. The average SR-FTIR spectrum obtained in these conditions is showed in Fig. 3. Also in this case in the diffraction limit regime, a good intensity ratio between the $\mathrm{CH}_{2}$ and the $\mathrm{CH}_{3}$ bands is obtained and indicates that these experiments are feasible and represent a very promising opportunity. Other characteristic bands are still detectable in the spectrum collected with a diffraction limited pinhole however, the $\mathrm{S} / \mathrm{N}$ ratio has to be improved.

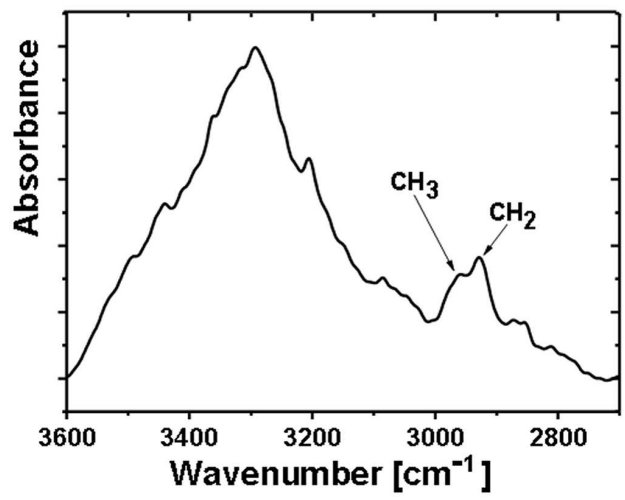

Fig. 3. SR-FTIR spectrum collected with a $5 \mu \mathrm{m}$ pinhole placed on the sample of a hyperplastic prostate tissue.

In the third set of experiments a large area of a hyperplastic prostate tissue was examined. The analysis was performed with a linear scan of $600 \mu \mathrm{m}$ at steps of $25 \mu \mathrm{m}$ and a total of 25 different points were measured. Spectra were collected at $8 \mathrm{~cm}^{-1}$ of resolution with a microscope aperture of $20 \mu \mathrm{m} \times 20 \mu \mathrm{m}$ and a total number of 100 scans. Figure 4 shows the photograph of the hyperplastic tissue sample with investigated line marked with dots which had been started on an epithelium area, crossed a gland and the stroma to end in a sec- ond gland. The histological complexity is characteristic of the hyperplastic prostate tissue structures. Therefore a SR-FTIR analysis at high spatial resolution, e.g., with a linear scan with a small step could give much more information about the local structure than any conventional FTIR bulk analysis.

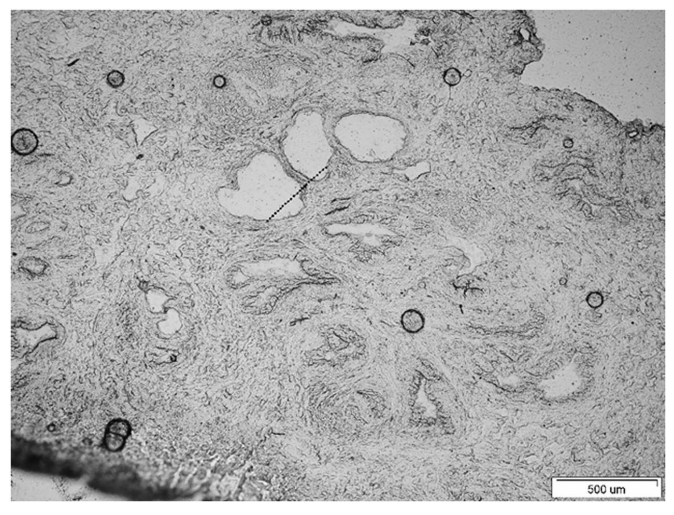

Fig. 4. Photo of the hyperplastic tissue sample where FTIR acquisitions at steps of $25 \mu \mathrm{m}$ over the distance of $600 \mu \mathrm{m}$ was performed.

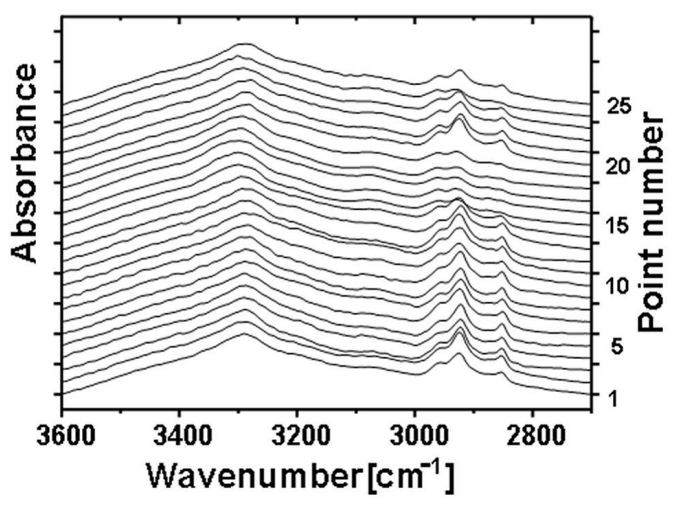

Fig. 5. Comparison of the SR-FTIR spectra collected in 25 points of the hyperplastic prostate tissue of Fig. 4.

Normalized FTIR absorbance spectra are compared in Fig. 5 vs. wavelength while the $\mathrm{CH}_{2} / \mathrm{CH}_{3}$ intensity ratio is shown in Fig. 6. FTIR spectra change during the scan and in particular the intensity ratio between $\mathrm{CH}_{2}$ and $\mathrm{CH}_{3}$ bands changes crossing different tissue components (see points between 15 and 19 vs. other points). The $\mathrm{CH}_{2} / \mathrm{CH}_{3}$ ratio covers the range from $1.25 \pm 0.15$ to $1.65 \pm 0.15$ and it is in agreement with previous determination [10].

\section{Discussion}

Based on the experiments performed one can see that the application of the SR-FTIR spectromicroscopy allowed to perform experiments with different apertures from fully opened aperture $-250 \mu \mathrm{m} \times 250 \mu \mathrm{m}$ (in this 
setup) down to $20 \mu \mathrm{m} \times 20 \mu \mathrm{m}$ and with small pinholes. In our configuration the $\mathrm{CH}_{2} / \mathrm{CH}_{3}$ intensity ratio could be detected with a high $\mathrm{S} / \mathrm{N}$ ratio. This combination of small aperture and pinhole made possible to achieve a subcellular spatial resolution. The use of $5 \mu \mathrm{m}$ pinhole (which is below $10 \mu \mathrm{m}$ ) allowed to achieve information from the area smaller than diffraction limit enabling to discriminate molecular states on the cell level.

FTIR spectra of tissues samples in different conditions: healthy, hyperplastic and cancerous stages, reveal differences that address the occurrence of chemical compositions changes in the examined samples. Moreover, the data showed the possibility of a reliable determination of the intensity ratio between the $\mathrm{CH}_{2}$ and the $\mathrm{CH}_{3}$ bands in the region $3000-2800 \mathrm{~cm}^{-1}$. In our specific case three different tissue sections can be recognized and characterized by a specific $\mathrm{CH}_{2} / \mathrm{CH}_{3}$ value similar as previously found $[10]$.

Looking at Fig. 6, the value of the ratio in the first region is significantly higher if compared to the following two. In second region the ratio is just slightly higher than one, probably addressing the characteristic value of healthy tissue. Again, it is in good agreement with previous observations [10]. In the last regions, spectra address values for the $\mathrm{CH}_{2} / \mathrm{CH}_{3}$ ratio larger than in the second but smaller than in the first region. We assign the occurrence of this difference to the different contents between the first and the second gland.

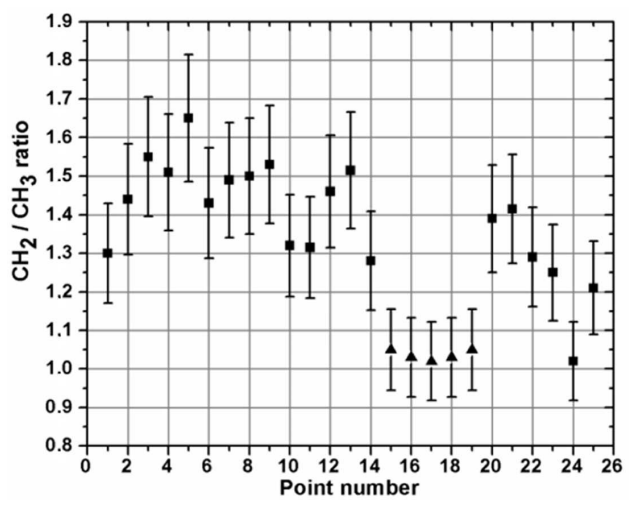

Fig. 6. The $\mathrm{CH}_{2} / \mathrm{CH}_{3}$ intensity ratio related to the spectra of Fig. 5 collected in the linear scan on the hyperplastic prostate tissue of Fig. 4.

This new opportunity, such as SR based microscopic FTIR spectroscopy is very important and stimulates new accurate FTIR experiments and in particular, a more accurate analysis of biological samples.

\section{Acknowledgments}

The authors acknowledge Prof. Jerzy Stachura and Prof. Tadeusz Cichocki of the Collegium Medicum of the Jagiellonian University for their assistance in tissue samples classification and preparation. Special thanks are given to Dr. Marek Gałka of the Gabriel Narutowicz Hospital who provided samples. This work was partly supported by Laboratori Nazionali di Frascati (Italy) under project TARI 06 Projects No. 16, 29, 48 contract No. RII3-CT-2004-506078.

\section{References}

[1] G.L. Carr, G.P. Williams, SPIE Conf. Proc. 3153, 51 (1997).

[2] N. Fujioka, Y. Morimoto, T. Arai, M. Kikuchi, Cancer Det. Prevent. 28, 32 (2004).

[3] A. Salman, R.K. Sahu, E. Bernshatain, U. Zelig, J. Goldstein, S. Walfisch, S. Argov, S. Mordechai, Vibrat. Spectrosc. 34, 301 (2004).

[4] E.O. Faolain, M.B. Hunter, J.M. Byrne, P. Kelehan, M. McNamara, H.J. Byrne, F.M. Lyng, Vibrat. Spectrosc. 38, 121 (2005).

[5] B.R. Wood, L. Chiriboga, H. Yee, M.A. Quinn, D. McNaughton, M. Diem, Gynecol. Oncol. 93, 59 (2004).

[6] C. Paluszkiewicz, W.M. Kwiatek, J. Mol. Struct. 565-566, 329 (2001).

[7] T. Yamada, N. Miyoshi, T. Ogawa, K. Akao, M. Fukusa, T. Ogasawara, Y. Kitagawa, K. Sano, Clinic. Cancer Res. 8, 2010 (2002).

[8] M. Huleihel, M. Karpasas, M. Talyshansky, Y. Souprun, Y. Doubijanski, V. Erukhimovitch, Vacuum 78, 557 (2005).

[9] E. Gazi, J. Dwyer, N.P. Lockyer, J. Miyan, P. Gradner, C.A. Hart, M.D. Brown, N.W. Clarke, Vibrat. Spectrosc. 38, 193 (2005).

[10] C. Paluszkiewicz, W.M. Kwiatek, A. Banaś, A. Kisiel, A. Marcelli, M. Piccinini, Vibrat. Spectrosc. 43, 237 (2007).

[11] W.M. Kwiatek, Postdoctoral Thesis (Habilitation), Polish Academy of Sciences, Kraków, Report No. 1928/PL (2003).

[12] W.M. Kwiatek, A. Banaś, K. Banaś, M. Podgórczyk, G. Dyduch, G. Falkenberg, M. Gajda, T. Cichocki, Acta Phys. Pol. A 109, 377 (2006).

[13] W.M. Kwiatek, A. Banaś, K. Banaś, A. Kisiel, G. Cinque, G. Falkenberg, Acta Phys. Pol. A 109, 383 (2006).

[14] W.M. Kwiatek, A. Banaś, K. Banaś, G. Cinque, G. Dyduch, G. Falkenberg, A. Kisiel, A. Marcelli, M. Podgórczyk, Spectrochim. Acta B, At. Spectrosc. 62, 707 (2007).

[15] M. Cestelli Guidi, M. Piccinini, A. Marcelli, A. Nucara, P. Calvani, E. Burattini, J. Opt. Soc. Am. A 22, 2810 (2005). 